\title{
Numerical modelling of infiltration profiles in the silt Tlemcen (Algeria)
}

\author{
Abdeldjalil Zadjaoui $^{1, a}$

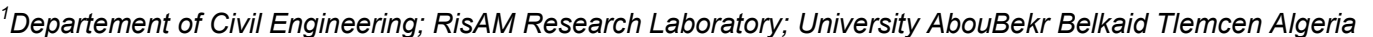

\begin{abstract}
It is obvious that for geotechnical knowledge of soil properties namely, suction-water content and water content hydraulic-conductivity relationship is essential to solve the problems associated with flow in unsaturated soils. The best way to evaluate these properties is to make direct measurements. However, the hydraulic-conductivity water content and suction-conductivity relationship are complex and it is often very difficult to measure for practical reasons, economic and spatial variability. Indeed, the used equipment is expensive and the tests are slow. Of more over the spatial variability of this property that makes the steps number necessary to characterize a site is relatively large. Furthermore, when this relationship is determined, usually it has only limited information and it is always desirable to extrapolate the experimental curve. Facing these difficulties, this given research targets computer tool to assess this relationship from other measurable parameters. In this paper, we used the software "flow line temperature" to achieve numerical and graphical simulations of the water flow in a soil initially unsaturated non-deformable modeling the evolution of the water content and suction in function of depth. The use of this software allowed us to make comparison with the experimental results [18] as well as a qualitative assessment of the drought penetrating in an unsaturated soil.
\end{abstract}

\section{Introduction}

The knowledge of soil properties, suction-water content and water content-hydraulic conductivity relationship is essential to solve the problems associated with flow in unsaturated soils. The best way to evaluate these properties is to make direct measurements. That during the hydraulic conductivity water content and suction conductivity relationships are complex and it is often very difficult to measure for practical reasons, economic and spatial variability. Indeed, the equipment used is expensive and tests are long, particularly because most of the measuring systems do not cover a large range of suction. Furthermore, the spatial variability of hydraulic properties makes the number of steps necessary to characterize a site relatively large. Also, once this relationship is determined, only limited information is available and it is always necessary to extrapolate the experimental curve. To face these difficulties, research is oriented towards to the use of digital tools in engineering practice. It is clear that the few necessary parameters in this case are easily measurable.

After a historical overview, concerning particularly the existing tools for identification, a theoretical overview of the infiltration problem is given. Subsequently, a description of the used experimental model and of the data needed for the simulations is exposed. An analysis using the software named "Flow line temperature" was performed with numerical simulations of the water flow in a column of a soil initially unsaturated and supposed to be non-deformable. The changes in water content and

\footnotetext{
${ }^{\mathrm{a}}$ Corresponding author: a.zadjaoui $@$ gmail.com
}

suction with respect to depth ad time were computed to allow comparisons with the experimental results. The findings in relation to the practice of geotechnical engineering are finally outlined.

\section{Background}

The description of water flow in unsaturated soils, described by the generalization of Darcy's law (1856) in unsaturated media, was mathematically summarized in the Richards' equation in 1931. The theory and basic assumptions are described in detail in the works of soil physics [11].

However, the problem of the non-uniqueness of the solution (humidification path and different drainage path) and the dependence of the conductivity with respect to the water content make it highly nonlinear. No analytical solution has been found to solve the Richard's equation.

Soil physicists have conducted several researches and proposed different resolution patterns $[6,1,5,8]$ under different initial and boundary conditions. All works provide numerical resolutions of the partial differential equations that governs the spatial and temporal changes of water content or pressure during a infiltration in a soil column. The major difference between these algorithms is their accuracy with respect to the mass balance error generated by the numerical approximations adopted.

Indeed, the use of these models often requires some information not always available in current engineering practice. This is why some software packages have 
recently been developed that allows easier use of flow model [20, 16, 15]. For example, [15] only discusses, in the package HYDRUS, various analytical expressions [17], while [20] includes in the new package SWIM different analytical expressions. Simunek and al. [15] introduced advanced procedures for two-dimensional flow and solute transport. The hypothesis of deformable medium was not mentioned in these references.

In this study one uses a new package that allows the one-dimensional simulation of a vertical flow in the laboratory or in situ $[3,4]$. The choice made partly relies on the easiness of use and on the existence of a dynamic display feature of moisture redistribution in the soil. Note that its use is free for academic [12].

\section{Theory and simulations description}

\subsection{Flow model}

According to [12], the model used to simulate the flow of water is established at macroscopic scale (a laboratory infiltration column) for a continuous and isotropic material. The flow is assumed to be vertical. The effects of air are neglected (assuming constant air pressure equal to atmospheric pressure) and the effects of chemical gradients (solute transport, chemical reaction with the ground) and temperature gradients are not considered. The simulation is then restricted to the vertical water transfer of water, in a homogeneous medium made up of several homogeneous layers. Modelling is based on four elements. To run, it requires:

The soil characteristics (general characteristics and water properties);

The initial water profile describing the amount of water contained in the soil $\left(\theta_{\mathrm{i}}\right)$

The algorithm solving the flow equation and the evolution over time of the boundary conditions applied to the soil (surface and deep boundary conditions).

If all data are available, each simulation will provide some information as follows:

- The simulated values of the water profile obtained by space discretization and by solving the flow equations discretized with respect to time

- The variables useful for understanding or validation of simulations launched.

\subsection{Distribution of water in the soil during the infiltration}

The distribution of the water in the ground at a given time is described by the water profile that depends on the physical properties of the soil and on the weather conditions. The infiltration of water at the surface causes a perturbation of the fluid profile that is characterized by 4 zones [11]. In the case of a homogeneous profile under constant infiltration, these zones are arranged as follows:

a. The surface saturation zone extends generally at a depth of $1 \mathrm{~cm}$; in the case of clay soil, this area may be subjected to a swelling phenomenon, which leads to very high water contents; b. The transmission area, almost saturated, is characterized by a very rapid decrease in water content to values of 70 to $80 \%$ saturation; this area becomes longer during infiltration. The saturation is apparently uniform in this zone which " $z_{\mathrm{s}}$ " is called depth of the wetting front.

c. The humidification area in which the water content changes rapidly with depth and time. It ends with the humidification brow; the distance between the edges at the surface is called the depth of the humidifying edge denoted " $\mathrm{z}_{\mathrm{f}}$ ".

d. The humidifying front is a region of a very high moisture gradient that represents a visible limit moisture penetration especially when the soil is initially dry.

Musy and Soutter [11] observe a transition zone between the vadose zone and the transmission zone. There is a zone of a few centimetres of depth through which the water content was reduced from saturation to a value characteristic of the transmission area. Subsequent research concerned the existence of both a zone of saturation and a distinct transition zone of the transmission area. An air trapping term explanation has been given, but it is possible that the saturating zone and the transition from a simple structure of soil disturbance which is unstable and subject to collapse in the vicinity of the surface by the action of the rain [7].

During infiltration, water penetrates the soil and gradually moves downwards by establishing successive water content profiles. The water distribution in the soil depends on the size of the grains. Periodic examination of the moisture profile during infiltration shows that the transmission area lengthens continuously, and that the humidification zone and the front move down. The front becomes less clear in depth. The suction gradient, initially high in a dry soil, decreases during infiltration to unit values.

\section{Application and comparison with experimental results}

\subsection{Application of the model}

To model infiltration in unsaturated soils, we used data obtained experimentally [18] on the Tlemcen silt with an initial water content of $13.8 \%$ and a permeability at saturation of $1.2 \times 10^{-7} \mathrm{~m} / \mathrm{s}$ for a hydraulic head of 120 $\mathrm{cm}$. The initial profile content was determined by using the filter paper technique. The model of Van Genuchten for the water retention curve and the Brooks-Corey model for hydraulic conductivity function were used.

\subsection{Comparison of water content profiles and moistening profiles}

The experimental work was performed at the Central Laboratory in Paris. The equipment used, the experimental protocol, the sample preparation technique and the acquisition system are detailed in [21]. Figure 1 shows the set of curves of evolution of $\theta=f(t)$ for 
different depths. In this case, we note that there is a shift in the experimental curves with respect to those obtained by simulation. This discrepancy (eg $\mathrm{z}=3 \mathrm{~cm} ; \mathrm{t}=7 \mathrm{~min})$ can be related to the fact that the initial conditions of laboratory water content obtained were difficult because the sample should have been left for more than 7 days to have a homogeneous water content profile. This finding is also observed at other depths.

Another important point is that the water supply is provided through a valve which provides once opened a large amount of water to the first ring placed just in contact. However, this difference is not very large compared to the time set for the specimen to reach saturation.

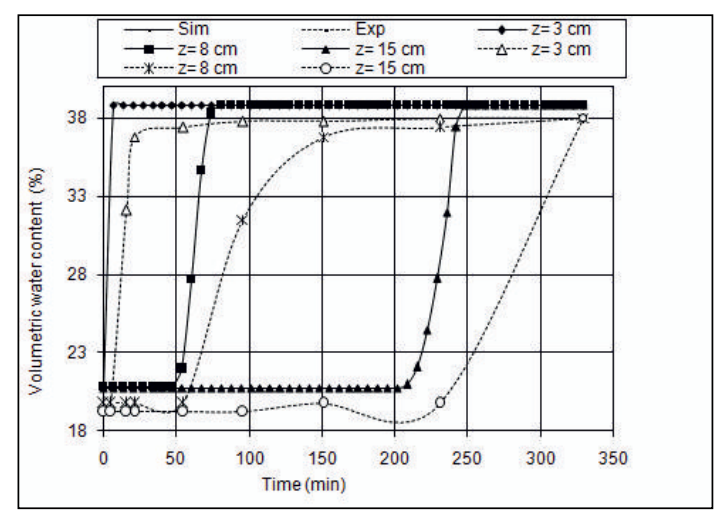

Figure 1. Comparison of levels of volumetric water.

The comparison of water profiles is shown in Figure 2. Figure $2 \mathrm{a}$ shows the profiles obtained experimentally while Figure $2 \mathrm{~b}$ represents the simulated profiles using the software "Flow line temperature".

It is clear that the time taken for the measurement of $\theta$ experimentally are different from those obtained by simulation. Only recent instants ( $t$ simulation) are those which give a difference in the water status in relation to the previous state. Nevertheless, it is thought likely that the choice of time $\Delta t$ has a considerable influence on the result.

It should be noted that there is a difference between $\mathrm{t}_{\text {fexp }}=330 \mathrm{~min}$ and $\mathrm{t}_{\text {fsim }}=265 \mathrm{~min}$ although $\theta_{\text {fexp }}=\theta_{\text {fsim }}=38 \%$.

This difference may be related to the diffusion phenomenon slowed in the laboratory during the vertical infiltration due to gravity effects.

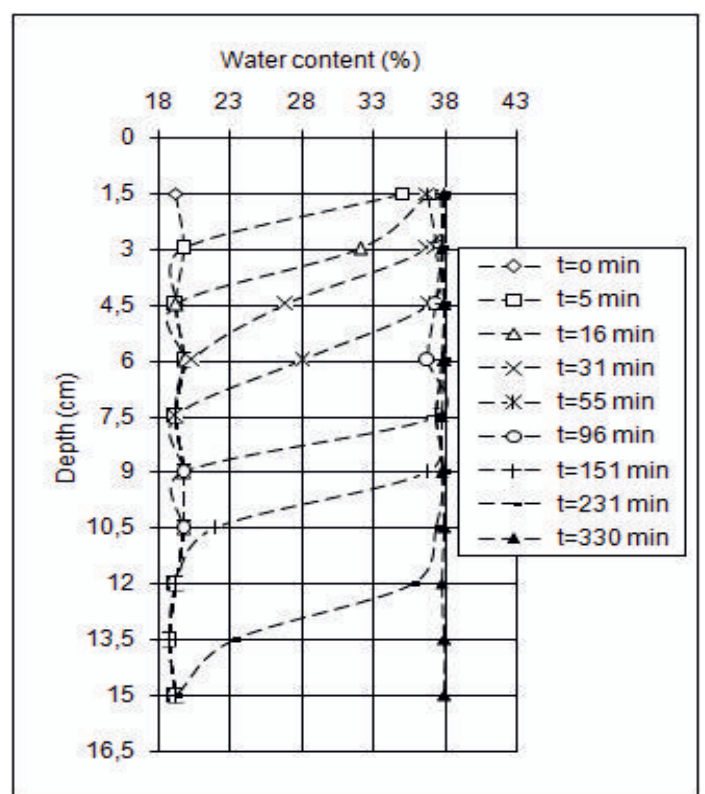

a) Experimental profile

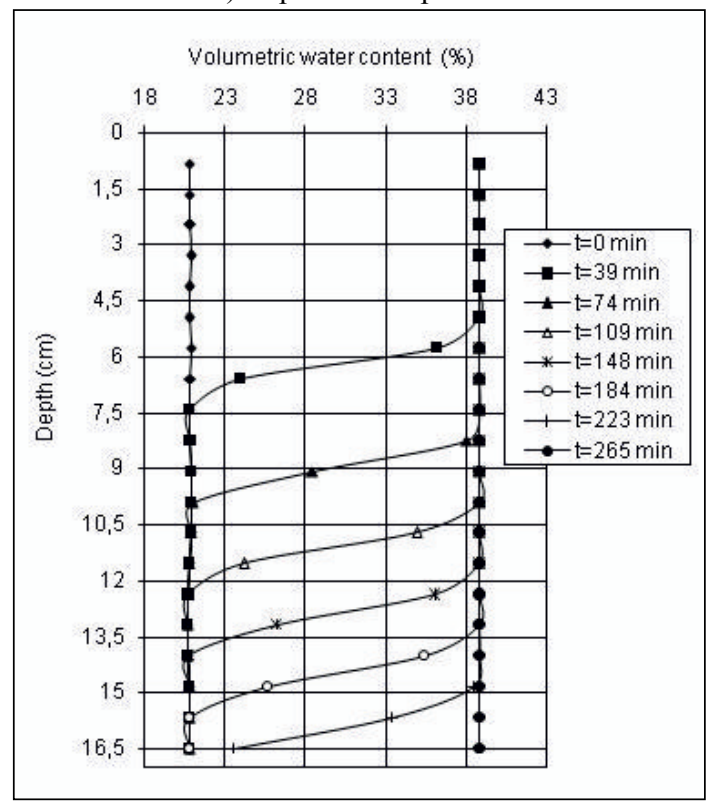

b) Simulation profile

Figure 2. Comparison between the contents profiles volumetric water experimentally obtained and those obtained by simulation.

\subsection{Comparison of cumulative infiltration}

Generally, infiltration increases monotonically until a limit corresponding to maximum infiltration. Figure 3 shows this increase in both the test and the simulation. One can see that the difference between simulation and experimental is increasing with larger periods of time.

One also observes that the saturation time is different for the two cases (a difference of 24.5\%). This is consistent with observations made by [9]. 


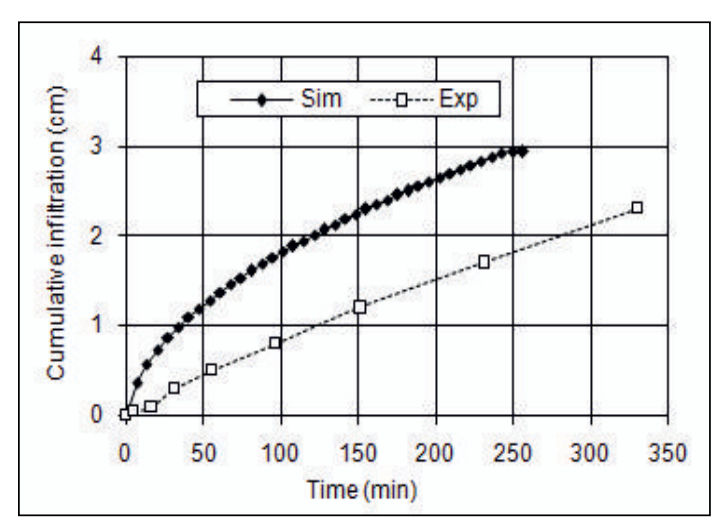

Figure 3: Comparison between the cumulative infiltration experimentally obtained and that obtained by simulation.

\subsection{Comparison of the rate of infiltration}

The description of the physical phenomenon of infiltration shows that the rate of infiltration is directly proportional to the humidity state of the soil. In the case of dry soil the rate of infiltration is relatively high.

Figure 4 is a graphic representation of the evolution of the infiltration rate with time. There is a decrease until the outflow corresponds to the complete saturation of the specimen.

The results obtained show a slight difference, that is larger for small values of time.

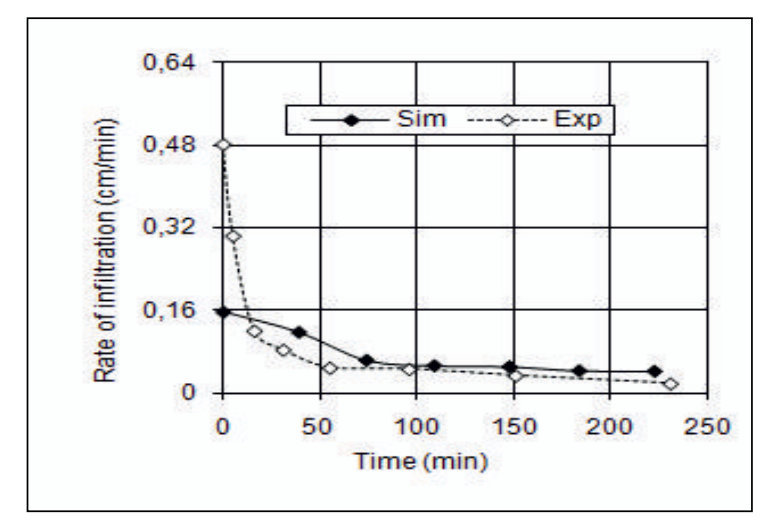

Figure 4. Comparing the rate of infiltration experimentally obtained with that obtained by simulation.

\subsection{Comparing the depth of the humidification front}

Figure 5 shows the evolution of the wetting front during infiltration. The observed trend is comparable to that of the cumulative infiltration. The progress of the front is directly related to the amount of water present in the sample. The difference between the experimental and the numerical range from $20 \%$ at $\mathrm{t}=31 \mathrm{~min}$ and $28 \%$ at $\mathrm{t}=$ $223 \mathrm{~min}$. The difference is smaller compared to that of the infiltration.

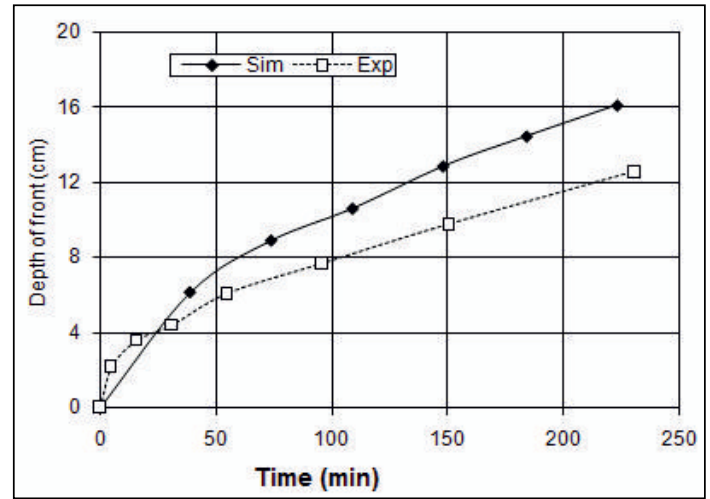

Figure 5. Comparing the depth of the humidity front obtained experimentally with that obtained by simulation.

\subsection{Comparison of the rate of advance of the front}

The evolution of the speed of the front with time is shown in Figure 6. This figure shows a difference between the experimental results and numerical which is especially important at the beginning. This difference may be related to the representativeness of the value of the volumetric water content $\theta$ and suction $\psi$ determined by the filter paper measuring depth (top part, bottom or middle of the inter-ring) [2].

But we note that the value of the progression speed during saturation is almost the same for both models. This explains the validity of Darcy's law and the exponential decay with respect to the root of time justifying the main assumption in the numerical solution procedure.

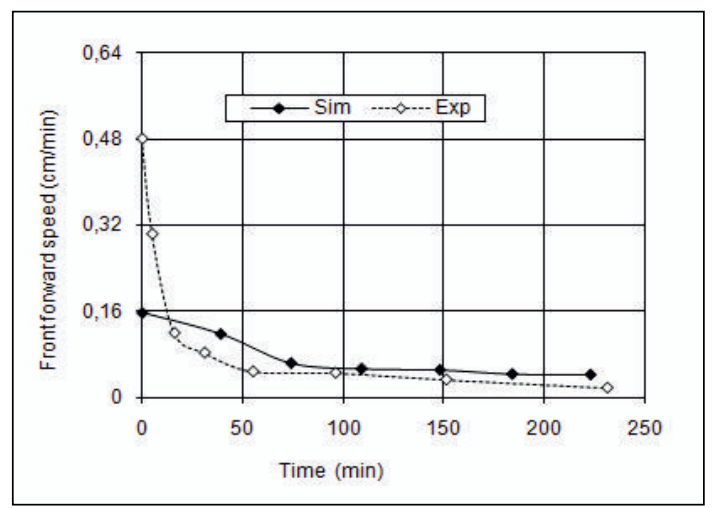

Figure 6. Comparison between the forward speed of the front and those obtained experimentally by simulation.

An indication of the variation as a function of drainage time given by simulation is shown in Figure 7 . At a height $\mathrm{h}_{0}$ of $120 \mathrm{~cm}$ a change is obtained at $\mathrm{t}=208$ $\min$. 


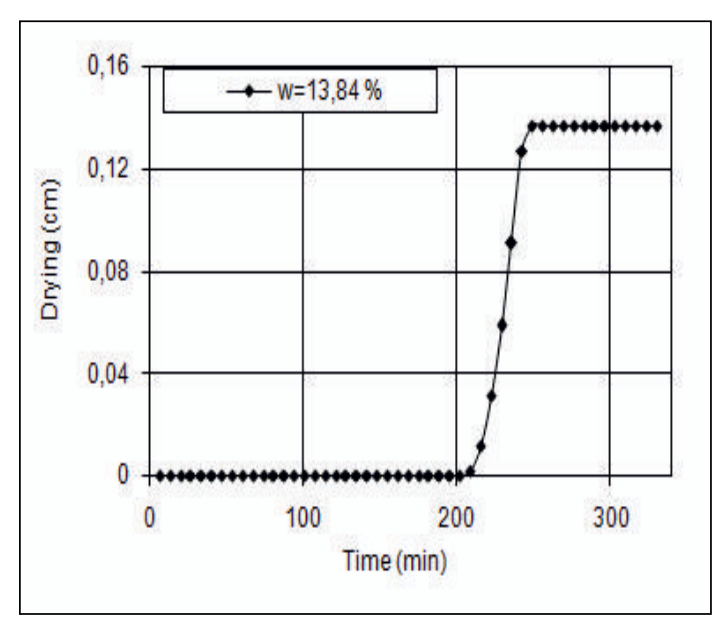

Figure 7. Cumulative drainage obtained by simulation for $\mathrm{h}_{0}=120 \mathrm{~cm}$.

\section{Conclusion}

The flow line temperature software is an interactive software that simulates the vertical flow of water in unsaturated soils. It is a convenient tool to predict the evolution of hydric soil profile with a wide range of possible boundary conditions, through a system of conventional numerical solution of the Richards equation. Flow must be characterized by water retention curves and hydraulic conductivity curves, the form may be selected from various analytical expressions of the literature. When parameters are not known, an inverse method is provided to estimate parameters from experimental data.

Comparing the numerical results with the experimental ones showed a slight difference that can be related to several causes including the mode of compaction used (static compaction), some errors in the measurements (error balance 0,001g) and also the influence of the filter papers measurements carried out during the infiltration.

Predicting the progression of the humidification front can be used together with the water content method for the prediction of swelling and shrink proposed by [19] and with the method of water profiles for the prediction of permeability of fine grained soils [18]. The ongoing work on models show whether this approach is good or not in the practice of engineering and geotechnical engineering.

\section{References}

1. Celia, M.A., Bouloutas, E.T., Zarba, R.L. A general mass-conservative numerical solution for the unsaturated flow equation. Wat. Res. Res. 26 (7), pp. 1483- 1496. (1990)

2. Didier G., Norotte V., Bentoumi O., Alimi-Ichola I. et Cazaux D. Protocole opératoire de mesure de la perméabilité en laboratoire- Cas des sols fins non saturés. Inst. Nat. Sci. App. Lyon. (1996)

3. Garnier, P. Détermination des caractéristiques hydrodynamiques de sols déformables par la méthode inverse. Ph.D. Dissertation, Nancy. (1996)
4. Garnier, P., Perrier, E., Angulo-Jaramillo, R., Baveye, P. Numerical model of 3-dimensional anisotropic deformation and 1-dimensional water flow in swelling soils. So. Sci. 162 (6), 410-420. (1997).

5. Gottardi, G., Venutelli, M. Richards: Computer program for the numerical simulation of one dimensional infiltration into unsaturated soil. Com. Geo. 19 (9), 1239-1266. (1993)

6. Haverkamp, R., Vauclin, M., Touma, J., Wierenga, P.J., Vachaud, G. A comparison of numerical simulation models for one dimensional infiltration. Soil Sci. Soc. Am. J. 41 (2), 285-294. (1977)

7. Hillel D. L'eau et le sol: Principes et processus physiques. Collection PEDASUP 5. (1988)

8. Ju, S.H., Kung, K.J.S., Mass types, element orders and solution schemes for the Richards equation. Com. Geos. 23 (2), 175-187. (1997)

9. Lafhaj Z., Samara M., Mania J., Sam R. et Hajnajib B. Mesures expérimentales de la perméabilité in situ des limons en zone urbaine au moyen de l'infiltromètre à double anneau et du perméamètre de Guelph. Coll. GeocityNet, Lille. (2005)

10. Leclerc, C., Le logiciel ECOUL, Mémoire de DESS Informatique. Université Henri Poincaré, Nancy. (1996).

11. Musy A. et Soutter M. Physique du sol. Presses Polytechniques et Universitaires Romande, Collection Génie de l'environnement, 6. (1991)

12. Perrier E., Garnier P. et Leclerc C. ECOUL: An interactive computer tool to study hydraulic behavior of swelling and rigid soils. Com. Geos. $281107-$ 1118. (2002)

13. Perrier, E., Garnier, P., Leclerc, C. Profile-scale simulation of water flow: A software package to visualize and estimate soil hydraulic properties effects, poster and demonstration, In: van Genuchten, M.Th., Leij, F.J., Wu, L. (Eds.), Proc. Int. Work. on Characterization and Measurement of the Hydraulic Properties of Unsaturated Porous Media. University of California, pp. 349-354. (1997)

14. Richards L.A. Capillary conduction of liquids through porous medium. Physic 1, pp.318-333. (1931)

15. Simunek, J., Van Genuchten, M.Th., Sejna, M., Using the HYDRUS-1D and HYDRUS-2D codes for estimating unsaturated soil hydraulic and solute. In: Van Genuchten, M.Th., Leij, F.J., Wu, L. (Eds.), Proceedings of the International Workshop on Characterization and Measurement of the Hydraulic Properties of Unsaturated Porous Media. University of California, pp. 1523-1536. (1999)

16. Van Dam, J.C., Hugen J., Wesseling J.G., Feddes R.A., Kabat P., van Walsum, P.E.V., Groenendijk, P., van Diepen, C.A., Theory of Swap version 2.0. Simulation of water flow, solute transport and plant growth in the soil-water-atmosphere-plant environment. Report 71, Water Resources, Wageningen University, Technical Document 45, Alterra Green World Research, Wageningen. (1997)

17. Van Genuchten, M.T. A closed form equation for predicting the hydraulic conductivity of unsaturated 
soils. Soil Science Society American Journal 44, 892-898. (1980)

18. Zadjaoui A., Bekkouche A. et Magnan J.P. La méthode des profils hydriques pour évaluer la perméabilité des sols fins ». Symposium International : Sécheresse et constructions. SEC'08, Marne-la-vallée, pp 287-294. (2008).

19. Briaud J.L. and Zhang X. Water content method for movement prediction of swell shrink soils.
Symposium International FondSup, Vol. 2. Magnan (éd.). (2004).

20. Verburg, K., Ross, P.J., Bristow, K.L. SWIMv2.1. User manual. Division Report, CSIRO Division of Soil, CSIRO Information Services, East Melbourne, Australia, 130. (1997).

21. Zadjaoui, A. Étude du transfert hydrique dans les sols non saturés : Échange sol - atmosphère. Ph.D. Dissertation, Tlemcen (Algeria). (2009). 